\title{
Bioengineering developments for paraplegic patients
}

\author{
J C Barbenel MSc PhD FRSE, Eur Ing J P Paul BSc PhD FRSE
}

Bioengineering Unit, University of Strathclyde, Glasgow, G1 ONW, Scotland.

\section{Introduction}

There has been a steady increase in the involvement of bioengineers in the rehabilitation of disabled people, including those who are disabled because of spinal cord injury. This involvement has largely been at a level which has led to the development of new or improved techniques and appliances. It has become apparent, however, that the bioengineer can, and should, play a management role in the organisation and management of rehabilitation services.

Theoretical investigations of spinal mechanics and the effect of trauma have grown both in number and complexity. In part this has been driven by improved methods of modelling and stress analysis of conventional engineering structures. Few of these studies have been experimentally validated and their clinical applicability appears, at present, to be marginal. We have, therefore, not discussed these theoretical studies but have concentrated on describing those areas of bioengineering which have had direct applications for the benefit of disabled people.

\section{Orthotics}

The development and introduction of thermoplastic materials for the construction of devices has had a major impact on orthotics, allowing appliances to be made more simply and cheaply. The resulting orthoses are generally lighter and have a better appearance than those made of more conventional engineering materials. The impact has, however, been rather limited in spinal cord injured patients who often require rigid bracing. The isotropic plastics used for the current devices are often too flexible for such bracing. The use of composites and anisotropic thermoplastics holds out considerable promise but further re- search and development is needed before they can be introduced for routine clinical use.

The major advance in orthoses themselves has been the development of devices which allow reciprocal gait. Several such devices have been developed and used, and more are at the development stage. The functional principles of all the devices are the same with the body being rigidly braced from mid-trunk to the feet with the ankles and the knees immobilised; the hips are prevented from moving into adduction but are allowed to both flex and extend. Walking is produced by moving the trunk forward while it is supported, eg by crutches or rollator, and then tilting the pelvis so that the trailing leg is lifted clear of the ground. The orthoses facilitate flexion of the hip and this allows the step to be taken. Prevention of adduction by a sufficiently rigid orthosis is important both to the ease with which the trailing leg clears the forward leg and to the possibility of using crutches.

Problems remain in the ease with which the devices can be donned or doffed by the user and the difficulty which wearers of the devices experience in standing up and sitting down.

\section{Functional electrical stimulation}

In spinal cord injury, although the neural connection between the brain and limbs is severed or partially severed, the distal segment of the nerve generally retains its blood supply and the neuromuscular unit retains its viability and responsiveness: although sometimes in unwanted ways. In particular, the tendon stretch reflex is generally present, and this results in spasm of the muscle in response to tendon stimuli. This may occur due to passive manipulation by a therapist at angular speeds above a critical 
value, and may sometimes produce a natural chain reaction resulting in clonus or a spasmodic cyclical movement of segments under the control of the affected muscles.

The responsiveness of the muscle allows external electrical stimuli to be used to produce useful contraction of muscle. These stimuli may be applied to the nerve or directly over the muscle belly or at different parts of the control loop. It has been found that the most effective results from stimulation may be produced using a carrier wave of $0.5-1 \mathrm{kHz}$ formed into approximately square pulses at $20 \mathrm{~Hz}$, usually a few milliseconds in duration. The shape of the pulse may be tailored to have a ramp at initiation and termination, and a refinement which has been found useful is to have a negative phase on the pulse over the quiescent period so that the total charge input in the active phase is balanced by the low level over the remainder of the phase. This is beneficial in terms of avoiding polarisation of the conducting tissues. At the present time most patients receiving functional electrical stimulation utilise surface electrodes. Fine wire electrodes have been used in certain research studies and are well tolerated by the few subjects involved, but there is a reluctance at the present time to adopt this procedure since it is invasive and has the potential of introducing infections round the wire on its passage through the skin. The Medical Research Council Neuroprosthesis Unit has developed implanted stimulators powered by radio control from an external loop aerial, although this is very much in the early development phase.

The major problem of functional electrical stimulation at the present time is that it is delivered on a simple on/off mode, and this does not allow for the graded muscular contraction which corresponds to normal functional use of the muscles. Studies are underway to get feedback from joint angle sensors or foot pressure sensors, but these systems still require much development work to be undertaken.

Patients selected for an FES programme may recently have had their accident or may be cases of long standing. It has been found that muscles may retain their excitability 10 to 12 years after the accident in some patients, and they thus have the potential to respond to FES. The first stage of treatment is to increase muscle bulk and strength by a training regimen consisting of 1-2 hours daily stimulation of the muscles against progressively increasing resistance for a period of 6-8 weeks. After such a period of training, it may be possible for these patients to stand from the seating position by stimulation of the quadriceps muscles. They retain their balance by the use of crutches and, indeed, no system sufficiently sophisticated to deal with balance has yet been developed. This ability to stand unaided may be the endpoint of the rehabilitation for many patients since it allows them to reach items on high shelves and has the benefit that periods of standing have been shown to improve circulation and bladder function. It is believed that standing will also help to reverse bone demineralisation.

A few patients who can achieve standing may be assisted to undertake a primitive form of walking. In this the quadriceps muscles are tensed when the leg is to bear body weight. To initiate a swing phase locomotion, the flexion response is initiated generally by stimulation of the peroneal nerve. The patient proceeds by successive stimulation of quadriceps and peroneal nerve on left and right limbs in turn, maintaining balance with the use of crutches.

The major problem in the long term application of FES is fatigue, and after 15-20 minutes stimulation, the contraction strength of a muscle may be reduced to about $10 \%$ of its maximum voluntary contraction level. Various strategies are being evaluated to avoid this phenomenon. The hybrid orthosis is one route. In this the patient receives an ankle-foot orthosis whose calf strap incorporates a load measuring transducer. The tension in the calf strap indicates when there is a tendency for the ankle to dorsiflex corresponding to load transmitted on the forefoot. This takes the knee into hyperextension and corresponds to a stable situation in which the patient is supported. Stimulation is applied to the quadriceps muscles only when there is risk of flexion as detected by the tension in the calf strap. In this way the muscles are 
stimulated only when necessary and the patient is enabled to stand for longer periods. An alternative method which has been considered is to use the 3 accessible parts of the quadriceps muscle and stimulate over a duty cycle of 2 seconds on and 4 seconds off, in vastus medialis, rectus femoris and vastus lateralis respectively. Thus the extending action at the knee may be delivered by the action of these muscles independently. It is realised that there may in the long term be problems, particularly from the patellofemoral joint, but present research is aimed at evaluating the technique in principle for possible subsequent clinical application.

In FES walking, as previously indicated, the swing phase is initiated by stimulation of the peroneal nerve. One problem with this is that the nerve system develops a 'habituation' and requires increasingly higher levels of stimulation to produce the flexion response or the response is erratic in its delivery. Stimulation at other sites has been tried and, although successful, was not accepted by the patients who have tried it. More recent studies have suggested that the habituation may be reversed by short duration pulses of $60 \mathrm{~Hz}$ stimulation at higher voltages.

In most systems of application of FES the control is an 'on-off' system. The so-called 'peroneal brace' may be prescribed for a patient with a mild hemiplegia following cerebral stroke if the functional loss is principally the ability to dorsiflex the foot. In this case the stimulation is applied during the swing phase of walking and a heel contact switch in the shoe is used to detect this. Simple crutch aided locomotion for the paraplegic patient is frequently controlled by push button switches on the handles of the crutch. Obviously this is a primitive control system being simply on an 'on-off' basis and involving a time lag due to the patient's assessment of the stage of the gait cycle. Studies are underway to develop more sophisticated control systems. These will be essential before multimuscle implanted stimulation units can be used. Since much of this work is at the development phase, there is much merit in utilising surface stimulation until appropriate control systems have been developed. It must be recognised however that for many of the patients the cosmetic improvement in leg shape, the physiological improvement in circulation and the ability to transfer between bed, chair and wheelchair may be the ultimate attainment. However, with the development of compact microprocessor systems it may be that the more challenging tasks may be achieved sooner than we expect.

\section{Pressure sore prevention}

There has been an increasing interest in pressure sore prevention by education research and development, and clinical service. Paraplegic and tetraplegic people, who are at particular risk of developing sores, have benefited from this activity.

The main biomechanical input has been in the development of improved support surfaces. In the case of beds this has largely been in the refinement of existing modes of support, particularly fluidised bead and low-loss air beds, rather than in any major innovation. There has, however, been considerable development of improved wheelchair support for the seated patient. This had led to relatively simple practicable means of making customised moulded or shaped matrix seats. Novel and improved materials have played an important role in the development of improved foam and fluid or gel cushions. The provision of improved seats and cushions have been accompanied by practical methods for measuring the interface pressures between the support surface and the patient which has allowed confirmation of the suitability of the cushion for the patients for whom they have been constructed.

Interest in, and development of, warning devices to remind the seated patient to move in order to relieve and redistribute the interface pressure seem to have undergone a steady decline. This may be due to the realisation that the patients who most need these devices are least likely to respond to the warnings. It may also reflect the more general understanding that devices are inadequate substitutes for sound rehabilitation and patient selfcare.

Functional electrical stimulation has also 
recently been used to produce pressure relieving movements but it is not yet possible to assess the usefulness and applicability of this technique.

\section{Patient assessment and management}

The development of improved appliances and method of treatment goes hand in hand with improved patient assessment, provision of devices and management. There remain major problems of patient assessment, with the requirement to select and supply the devices which are most appropriate to the patient's abilities and needs. It is most important that the bioengineer plays a role in this assessment as part of a group of people with broad medical and paramedical expertise - the multidisciplinary clinic team. The organisation and management of the provision and maintenance of devices have emerged as an area of growing importance in the application of clinical bioengineering.

The most important lesson which has been learnt by bioengineers involved in the rehabilitation of spinal cord injured patients is that the provision of the device is not the endpoint of their involvement but the start of a long term process of patient care and management. 